\title{
Commentary Attributable mortality of Acinetobacter baumannii: no longer a controversial issue
}

\author{
Matthew E Falagas ${ }^{1,2,3}$ and Petros I Rafailidis 1,3
}

\author{
${ }^{1}$ Alfa Institute of Biomedical Sciences, Athens, Greece \\ ${ }^{2}$ Department of Medicine, Tufts University School of Medicine, Boston, Massachusetts, USA \\ ${ }^{3}$ Department of Medicine, Henry Dunant Hospital, Athens, Greece
}

Corresponding author: Matthew E Falagas,m.falagas@aibs.gr

Published: 31 May 2007

This article is online at http://ccforum.com/content/11/3/134

(c) 2007 BioMed Central Ltd
Critical Care 2007, 11:134 (doi:10.1186/cc5911)

from the United States of America [6], Israel [7], Australia [8] and Korea [9] have provided their findings regarding the effect of $A$. baumannii infections in patients treated in the intensive care unit setting and also on medical and surgical wards. Another recent study from Israel compared $A$. baumannii bacteremia with Klebsiella pneumoniae bacteremia [10]. We summarize the findings of these five newer studies in Table 1.

The result of the main analysis of the latter study showed that A. baumannii remained significantly associated with mortality after adjustment for other important risk factors was performed [10]. Subgroup analyses in the same study again confirmed the association of $A$. baumannii with increased mortality in those patients not mechanically ventilated in the 30 days prior to bacteremia (multivariate analysis: odds ratio $=3.98,95 \%$ confidence interval $=1.25-12.62$ ) and in those patients not presenting with septic shock (odds ratio $=$ $4.62,95 \%$ confidence interval $=1.74-12.22$ ).

Infections due to Acinetobacter baumannii have been frequently considered by clinicians and researchers not to be associated with considerable mortality [1]. Indeed, A. baumannii has been placed in the list of low-virulence pathogens [2]. These beliefs have generated relative widespread beliefs among members of the medical community that this microorganism is not a cause of considerable mortality in hospitalized patients, and have generated controversy on the issue of attributable mortality of $A$. baumannii infections $[1,3]$. We recently performed a systematic review of the literature of cohort and case-control studies that focused on the issue [4]. The reviewed data suggest that infection with $A$. baumannii is associated with considerable mortality. In addition, we provided data regarding the impact of inappropriate empirical treatment of $A$. baumannii infections [5].

During the first 3 months of 2007, four new studies were added to the relevant literature. Specifically, investigators
In addition, in a recent letter to the editor, the summary findings of an older case-control study was reported that compared patients who were colonized or infected with multidrug-resistant $A$. baumannii with patients who were colonized or infected with multidrug-resistant Pseudomonas aeruginosa. Increased mortality was noted in the $A$. baumannii group (a statistically significantly result), even after adjusting for the stay in the intensive care unit [11].

Added to the findings of the previously available studies, we believe that these new data clearly show that infection of A. baumannii is associated with considerable mortality, even after adjustment for important potential confounders such as disease severity and the effect of the empirical antimicrobial treatment. While one could theoretically always argue that a specific risk factor may not have been included in the matching process of case-control and cohort studies (and thus differences in mortality may be attributed to this particular 


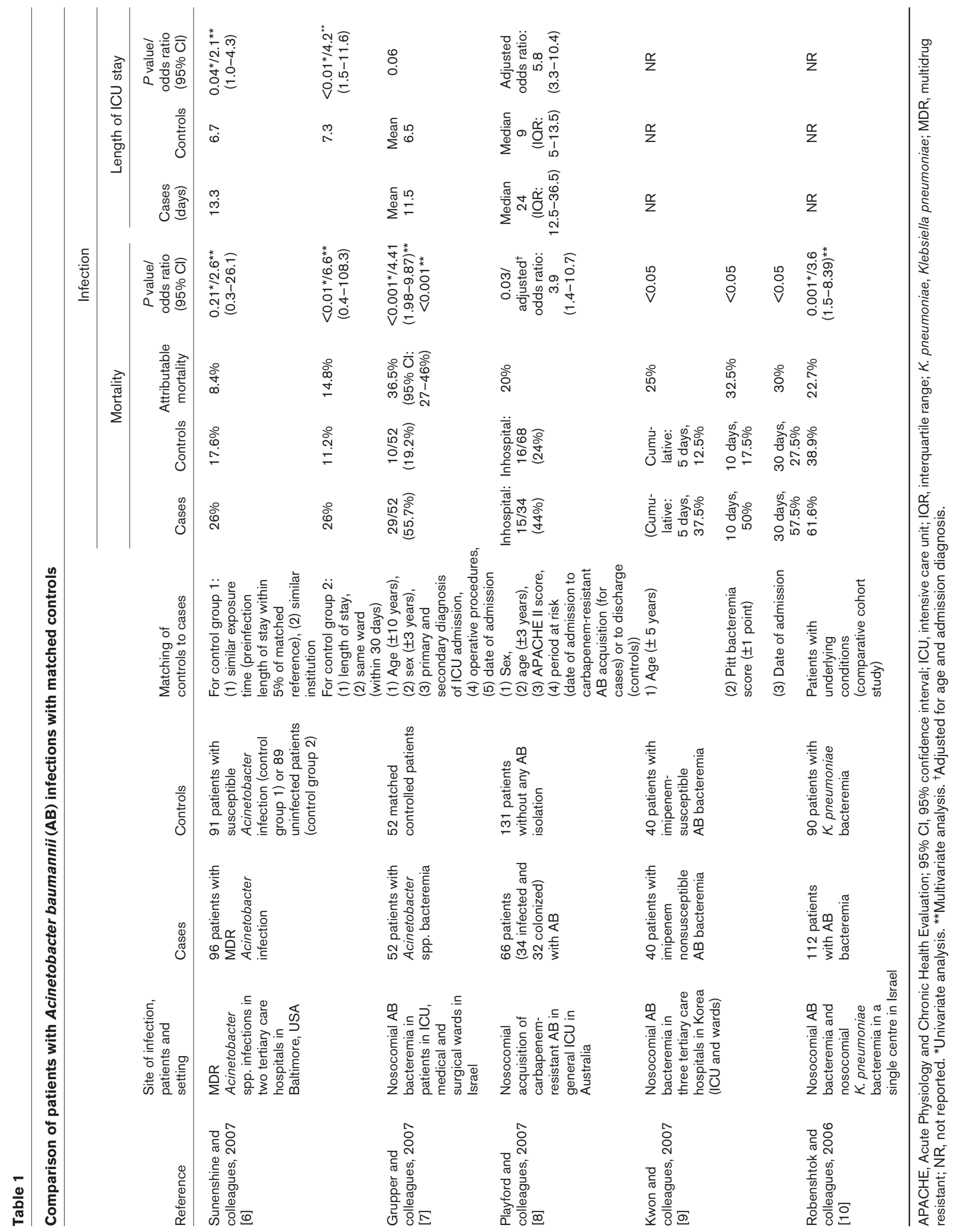


nonexamined risk factor), one has simultaneously to acknowledge the hazards of adjusting to all possible factors [12].

Someone may rightfully ask why not all relevant studies showed increased mortality of patients with $A$. baumannii infections compared with controls without such infections. There are various explanations for these apparently controversial findings. These explanations may include differences in patient populations, methodological characteristics of the studies, proportions of patients who received appropriate empirical treatment, as well as factors associated with the pathogen itself, including genetic factors that lead to differences in virulence.

If $A$. baumannii infection was not an independent contributor to increased mortality, then inappropriate treatment should not have any major effect on the outcome of patients. In an older study, mortality due to $A$. baumannii bacteremia treated with inappropriate antibiotics was $7.6 \%$ in excess (although not a statistically significant difference) compared with that of patients treated with appropriate antibiotics [13]. The finding of this study has to be interpreted considering that no excess mortality was found for any other bacteremia (possibly due to the small number of patients) except for bacteremia due to coagulase-negative Staphylococci [13]. In a study by our group, inappropriate treatment of $A$. baumannii bacteremia was associated with considerably increased (25.8\%) mortality compared with that of patients treated with appropriate antibiotics - significance reached a trend $(P=0.1)$ since the total study population was small (40 patients) [5].

In the recently published study by Kwon and colleagues, a $44.5 \%$ increased mortality was noted in the group of patients with imipenem nonsusceptible $A$. baumannii bacteremia treated with inappropriate antibiotics compared with those patients with imipenem nonsusceptible $A$. baumannii bacteremia who received appropriate treatment $(P=0.007)$ [9]. Moreover, when 30-day mortality was examined in the total study population of cases and controls, a $43.7 \%$ excess mortality was noted when $A$. baumannii bacteremia was treated with inappropriate versus appropriate antibiotic therapy $(P<0.001)$ [9]. Also, in a study regarding risk factors for mortality related to nosocomial pneumonia due to various Gram-negative, Gram-positive and fungal pathogens, $A$. baumannii was the second most commonly cultured pathogen of 132 patients. In this study $A$. baumannii was significantly more predominant in nonsurvivors than in survivors $(13.6 \%$ versus $5.1 \%, P=0.04)$. Of the nonsurvivors, $85.9 \%$ received inappropriate therapy inappropriate antibiotic therapy was independently associated with mortality (odds ratio $=14.5$, 95\% confidence interval $=5.08-41.44)$ in the study [14].

We believe that a fair interpretation of the available evidence, especially in light of the recently published findings, suggests that attributable mortality due to $A$. baumannii is no longer a controversial issue. The attention of the scientific community with interest in this pathogen should therefore be directed to the development and introduction of new antimicrobial agents effective against multidrug-resistant and pandrug-resistant A. baumannii, as well as to the implementation of infection control measures that may help control the evolving problem of $A$. baumannii infections that have taken epidemic dimensions in several parts of the word, especially in critically ill patients.

\section{Authors' contributions}

MEF had the idea for this commentary. PIR extracted the data for the relevant studies. Both authors contributed to writing the commentary and approved its final version.

\section{Competing interests}

The authors declare that they have no competing interests.

\section{References}

1. Fournier PE, Richet $\mathrm{H}$ : The epidemiology and control of Acinetobacter baumannii in health care facilities. Clin Infect Dis 2006, 42:692-699.

2. Harbarth S, Nobre V, Pittet D: Does antibiotic selection impact patient outcome? Clin Infect Dis 2007, 44:87-93.

3. Falagas ME, Kopterides P, Siempos II: Attributable mortality of Acinetobacter baumannii infection among critically ill patients [letter]. Clin Infect Dis 2006, 43:389; author reply 389-390.

4. Falagas ME, Bliziotis IA, Siempos II: Attributable mortality of Acinetobacter baumannii infections in critically ill patients: a systematic review of matched cohort and case-control studies. Crit Care 2006, 10:R48.

5. Falagas ME, Kasiakou SK, Rafailidis PI, Zouglakis G, Morfou P: Comparison of mortality of patients with Acinetobacter baumannii bacteraemia receiving appropriate and inappropriate empirical therapy. J Antimicrob Chemother 2006, 57:12511254.

6. Sunenshine $\mathrm{RH}$, Wright MO, Maragakis LL, Harris AD, Song $X$, Hebden J, Cosgrove SE, Anderson A, Carnell J, Jernigan DB, et al.: Multidrug-resistant Acinetobacter infection mortality rate and length of hospitalization. Emerg Infect Dis 2007, 13:97103.

7. Grupper M, Sprecher H, Mashiach T, Finkelstein R: Attributable mortality of nosocomial acinetobacter bacteremia. Infect Control Hosp Epidemiol 2007, 28:293-298.

8. Playford EG, Craig JC, Iredell JR: Carbapenem-resistant Acinetobacter baumannii in intensive care unit patients: risk factors for acquisition, infection and their consequences. J Hosp Infect 2007, 65:204-211.

9. Kwon KT, Oh WS, Song JH, Chang HH, Jung SI, Kim SW, Ryu SY, Heo ST, Jung DS, Rhee JY, et al:: Impact of imipenem resistance on mortality in patients with Acinetobacter bacteraemia. J Antimicrob Chemother 2007, 59:525-530.

10. Robenshtok E, Paul M, Leibovici L, Fraser A, Pitlik S, Ostfeld I, Samra Z, Perez S, Lev B, Weinberger M: The significance of Acinetobacter baumannii bacteraemia compared with Klebsiella pneumoniae bacteraemia: risk factors and outcomes. $J$ Hosp Infect 2006, 64:282-287.

11. Gkrania-Klotsas E, Hershow RC: Colonization or infection with multidrug-resistant Acinetobacter baumannii may be an independent risk factor for increased mortality. Clin Infect Dis 2006, 43:1224-1225.

12. Schwaber MJ, Carmeli Y: Antimicrobial resistance and patient outcomes: the hazards of adjustment. Crit Care 2006, 10:164.

13. Zaragoza R, Artero A, Camarena JJ, Sancho S, Gonzalez R, Nogueira JM: The influence of inadequate empirical antimicrobial treatment on patients with bloodstream infections in an intensive care unit. Clin Microbiol Infect 2003, 9:412-418.

14. Lee SC, Hua CC, Yu TJ, Shieh WB, See LC: Risk factors of mortality for nosocomial pneumonia: importance of initial anti-microbial therapy. Int J Clin Pract 2005, 59:39-45. 